\title{
Chine. Traditions populaires instrumentales
}

Enregistrements : Institut de musique chinoise, Académie des arts de Chine, Beijing (1950-1986), Stephen Jones (1986-1992) et autres (1930, 1960), 1995

\section{Lucie Rault-Leyrat}

\section{OpenEdition}

Journals

Édition électronique

URL : http://journals.openedition.org/ethnomusicologie/1313

ISSN : 2235-7688

Éditeur

ADEM - Ateliers d'ethnomusicologie

Édition imprimée

Date de publication : 1 décembre 1996

Pagination : 356-358

ISBN : 978-2-8257-0559-9

ISSN : 1662-372X

Référence électronique

Lucie Rault-Leyrat, « Chine. Traditions populaires instrumentales », Cahiers d'ethnomusicologie [En ligne], 9 | 1996, mis en ligne le 05 janvier 2012, consulté le 01 mai 2019. URL : http:// journals.openedition.org/ethnomusicologie/1313

Ce document a été généré automatiquement le 1 mai 2019.

Tous droits réservés 


\section{Chine. Traditions populaires instrumentales}

Enregistrements : Institut de musique chinoise, Académie des arts de Chine, Beijing (1950-1986), Stephen Jones (1986-1992) et autres (1930, 1960), 1995

\section{Lucie Rault-Leyrat}

\section{RÉFÉRENCE}

Chine. Traditions populaires instrumentales.Enregistrements : Institut de musique chinoise, Académie des arts de Chine, Beijing (1950-1986), Stephen Jones (1986-1992) et autres $(1930,1960)$; textes de présentation : Stephen Jones. 2 CDAIMP XXXVI-XXXVII (Archives internationales de musique populaire, Musée d'ethnographie, Genève). VDE 822-823, 1995.

1 A travers le choix des enregistrements présentés dans ces deux CD, Stephen Jones opte pour une exploration de ce qui, dans la musique chinoise dite populaire, a perduré au cours des bouleversements sociologiques de ces dernières décennies. C'est tout à la fois un tour d'horizon historique et thématique qui est ainsi proposé, qui veut écarter systématiquement de son champ d'investigation d'une part les genres favorisés par des options politiques et, d'autre part, ceux qui seraient marqués par une vénérabilité trop fossilisée.

2 Ce qui est ici mis en lumière se veut représenter une expression vivante et, au sein de celle-ci, la musique instrumentale, parmi d'autres traditions en vigueur, telles que le chant populaire, l'opéra ou la ballade. Le parti ainsi pris de musique vivante jette un pont par dessus les gouffres de silence et de néantisation creusés par la révolution culturelle, réactualisant des enregistrements anciens et les mettant en parallèle avec des documents plus récents de la même veine. Il ressort de ce principe la preuve tangible de l'existence d'une tradition de musique instrumentale vivace, enracinée dans la vie sociale, transmise 
avec tout à la fois un souci de continuité et de modernité. Comme toutes les traditions vivantes, ces musiques maintiennent leur originalité tout en s'alimentant des apports extérieurs ponctuels et en les intégrant à leur trajectoire évolutive.

Parmi les enregistrements proposés, classés selon deux zones géographiques principales (CD 1: Chine du Nord, CD 2: Chine du Sud), on se trouve confronté à un contexte sociologique où la musique se transmet au sein du noyau familial ou dans le cadre d'associations régionales, religieuses ou laïques. Que ce soit à Beijing même ou dans le reste du Hebei, au Shanxi, au Liaoning, au Shandong ou au Shaanxi, les exemples musicaux pré- ou post-révolutionnaires rendent comptent d'une continuité dans l'activité des associations musicales; le rôle de celles-ci, déterminant dans la musique chinoise d'aujourd'hui, parvient à combler les lacunes imputables aux événements politicoéconomiques: si telle tradition instrumentale mélodique est tombée en désuétude, des ensembles shengguan (orgues à bouche-chalumeaux) palient cette déficience en pratiquant les rituels qui les sous-tendaient. Que ce soient des musiques liées aux cérémonies de pluie (pl. 1 : Shuilong yin, «Le cri du Dragon d'eau »; pl. 4 : Batiaolong, « Les huit Dragons») ou d'autres musiques empreintes de sacré (pl. 2: Xiao huayan, «Petit Avatamsaka »), ainsi que la longue suite bouddhique Pu'an zhou, «Le mantra de Pu'an», (pl. 5) et les suites cérémonielles de «musique assise» (pl. 6-7), on retrouve, se faisant écho, des airs enregistrés dans les années cinquante, où se fit jour une prise de conscience de l'héritage musical que représentait la musique populaire et où l'Institut de recherche musicale de l'Académie des arts de Beijing réalisa sur le terrain d'importantes collectes, et d'autre part, des enregistrements plus récents qui rendent compte de la survivance et de la richesse de ce fonds populaire.

4 Le deuxième $\mathrm{CD}$, consacré à la Chine du Sud, témoigne lui aussi de la transmission des enseignements qui ont survécu à la révolution culturelle, notamment dans l'art des percussions (pl. 1-2: Shifangu et Shifanluogu), la permanence des genres Nanguan et Shiyin, les styles civils et martiaux (pl. 3-4), la musique de Chaozhou, dans tout le raffinement de ses ensembles de chambre à cordes (pl. 5-6: Liuyao jin, «Elle dont la taille est si fine»; Liuqing niang, « Dame Liuqing ») ; quant au genre martial propre à Chaozhou, il est illustré ici par un arrangement de Fan Lihua po zhen, «Fan Lihua traverse les lignes ennemies », où se combinent le style " gong et tambour » et les mélodies ditao, "Suites pour flûte ». Enfin, la musique cantonnaise est illustrée par un enregistrement mémorable réalisé vers 1930 ( Shuangsheng lian, "Double expression d'amertume», pl. 8) où divers instruments occidentaux se mêlent à l'instrumentarium traditionnel, révélant une hybridité de genres propre au contexte social de l'époque, qui s'est perpétuée sous forme d'une musique légère citadine se voulant représentatrice de la musique populaire chinoise, mais allant à l'encontre de celle-ci, comme le prouvent les musiques non falsifiées et vivantes écoutées sur les plages précédentes.

5 Ce tour d'horizon des traditions populaires instrumentales de la Chine se fait avec un plaisir d'autant plus grand qu'on a l'impression intense de découvrir un trésor de musiques vivantes, reliées à un contexte social authentique, qui se déploient dans la trame de coutumes et de cultures dont l'ancienneté se renouvelle sans jamais s'épuiser. Les campagnes chinoises semblent avoir traversé les épreuves que leur a infligées l'Histoire avec beaucoup de résistance et de bonheur, plus proches des valeurs agricoles dans leur pérennité, moins sujettes aux brusques changements qu'ont causé les mots d'ordre aux classes lettrées. Notons également la sauvegarde des enseignements grâce au relais assuré par les milieux taoistes et bouddhistes. 
6 A travers les enregistrements que réunit ce disque, on constate que ce sont les campagnes et leurs ensembles dits populaires qui ont perpétué la conservation des normes propres à la musique chinoise et, paradoxalement, son classicisme. 